\title{
EDITORIAL
}

\section{U nidades de tratamiento del ataque cerebrovascular (UTAC) en Chile}

\author{
Stroke Units in Chile
}

\begin{abstract}
Stroke is a major public health problem in Chile, with an incidence similar to that of many industrialized nations, it accounts for $10 \%$ of all deaths and ranks $5^{\text {th }}$ in number of healthy years of life lost to a disease. Organized inpatient care for stroke patients has been shown in systematic reviews of randomized clinical trials to be effective in preventing death, disability and institutional care, without increasing length of stay compared to usual care. Organized stroke unit care is provided by multidisciplinary teams that exclusively manage stroke patients in a dedicated ward (stroke ward) of which there are various possible models; acute stroke units which accept patients acutely but discharge early (usually within 7 days). This could include an "intensive" model of care with continuous monitoring and high nurse staffing levels; comprehensive (i.e. combined acute and rehabilitation) stroke units which accept patients acutely but also provide rehabilitation for at least several weeks if necessary. The way in which stroke units affect outcome is through reduction of complications of immobility and probably non specific neuroprotection. Stroke units should deliver high quality evidence based interventions to all eligible patients and should maintain high standards of care through staff training, guidelines and protocols, audit and quality assurance. Given that stroke is one of the fifty six health priorities in the health reform, inpatient stroke care should be delivered through stroke units organized in hospitals throughout Chile.
\end{abstract}

L os ataques cerebrovasculares (ACV) están incluidos en las llamadas epidemias emergentes de las enfermedades crónicas no transmisibles. En Chile son la segunda causa específica de muerte desde hace más de 10 años y constituyen la primera causa de hospitalizaciones sobre los 65 años ${ }^{1}$. En 2003 egresaron 19.700 pacientes con diagnóstico de $\mathrm{ACV}^{1}$. En el estudio de carga de enfermedad, fueron la quinta causa de años de vida ajustados por discapacidad (AVISA), mostrando su relevancia como problema de salud del adulto $^{2}$.

Correspondencia a: Dr. Pablo M. Lavados. Clínica Alemana de Santiago. Av. Manquehue Norte 1401, 6o piso. Vitacura, Santiago. Fono-Fax: 2101061. E-mail: pablolavados@yahoo.com
La incidencia del ACV en Chile ha sido estudiada recientemente. El estudio PISCIS, realizado en Iquique, encontró una tasa de ACV total de 168,4 casos por 100.000 habitantes ajustados a la población chilena, con lo que se esperaría a nivel nacional alrededor de 27.000 casos anuales ${ }^{3}$. De los pacientes que sobreviven a los 6 meses sólo $47 \%$ lo hacen de manera independiente, $\mathrm{y}$ $63 \%$ necesita algún tipo de ayuda, lo que significa un importante impacto económico y social para la familia y el país.

Uno de los problemas en el manejo de estos pacientes, fue por muchos años la falta de tratamientos efectivos. Actualmente existe sólida evidencia de que al menos tres intervenciones son eficaces en la reducción de muerte o dependencia a 6 meses, en pacientes que han sufrido un ACV 
agudo: 1) El uso de aspirina en los primeros 14 días después de un infarto cerebral; 2) La trombólisis intravenosa en infartos cerebrales con menos de 3 horas de evolución y 3) la hospitalización en una unidad especializada en el manejo de estos pacientes, cualquiera sea el tipo de ACV agudo ${ }^{4}$.

Las unidades especializadas en el tratamiento del ACV agudo o UTACs (Stroke Units, en inglés) se definen como un área clínica geográficamente limitada, con un equipo multiprofesional coordinado, que provee todos los cuidados médicos y de rehabilitación que el paciente requiera. El equipo consta de médico, enfermera, kinesiólogo, fonaudiólogo, terapeuta ocupacional y neuropsicólogo.

Existe evidencia tipo I, que permite hacer una recomendación grado A respecto a la efectividad de las UTACs. Un metaanálisis de la Colaboración Cochrane demuestra que el número necesario de pacientes por tratar en una UTAC, para prevenir una muerte, es de 33, el número necesario por tratar para que un paciente vuelva a vivir a su casa 20, el número necesario por tratar para prevenir un paciente dependiente es de 20. La hospitalización en una UTAC reduce los días de estada y el efecto se mantiene a los 10 años de seguimiento. Se benefician pacientes de todas las edades, ambos sexos y todos los grados de severidad ${ }^{5}$.

La organización de la UTAC puede ir desde una unidad que provea atención en los primeros siete días con monitorización intensiva no invasiva (menos evidencia), hasta unidades que provean monitorización fisiológica básica y rehabilitación por dos a cuatro semanas (mayor evidencia) ${ }^{5}$.

Los pacientes con ACV agudo presentan un gran número de posibles anormalidades fisiológicas, neurológicas y complicaciones generales que deben y pueden ser sospechadas, prevenidas, detectadas y tratadas eficazmente para optimizar el desenlace. La omisión de estos cuidados probablemente anula los beneficios de cualquier intervención farmacológica 0 procedimiento agudo. Cabe destacar que los factores que determinan la efectividad de una UTAC no son la realización de trombólisis, ni la monitorización fisiológica intensiva.

Mellado y cols nos presentan en este número de la Revista Médica de Chile las características de los pacientes con ACV agudo hospitalizados en un modelo particular de UTAC; localización en un área geográficamente definida; monitorización no invasiva; médico neurólogo dedicado y alta dotación de enfermería; acceso a rehabilitación externa e imágenes del cerebro; y estadías cortas ${ }^{6}$. El modelo más estudiado y que ha demostrado tener beneficios es el modelo exhaustivo, en el cual se da énfasis tanto al manejo agudo como a la rehabilitación precoz, y las estadías son más prolongadas. La forma en la cual las UTACs ejercen sus beneficios no está determinada por una única intervención. Probablemente, es más bien el modelo de organización en torno a las verdaderas necesidades de un paciente con ACV agudo: énfasis en su rehabilitación precoz y sostenida; disminución de complicaciones debidas a inmovilidad; evaluación de disfagia y prevención de neumonía aspirativa; evitar la hipotensión, hiperglicemia, hipoxemia, hipercapnia e hiponatremia.

Los ACV forman parte de las 56 prioridades de salud del plan AUGE y dado la evidencia de eficacia de las UTACs, la hospitalización del mayor número posible de pacientes con ACV agudo en UTACs debiera ser una garantía explícita (GES) y un avance para Chile. Esto significará conformar equipos profesionales que incluyan médicos neurólogos dedicados y capacitados, enfermeras, kinesiólogos, fonoaudiólogos, terapeutas ocupacionales y psicólogos. El trabajo de este equipo multiprofesional debe proveer un ambiente en el cual las actitudes, tratamientos e intervenciones eficaces, sean administradas a todos los pacientes susceptibles de beneficiarse con ellas. Para asegurar el mejor desenlace funcional posible, el equipo debe actuar de acuerdo a protocolos, guías de práctica clínica, procesos clínicos integrados sujetos a control de calidad y auditoría ${ }^{4}$. Tengamos en claro entonces que el desafío que tenemos por delante no es menor.

\section{Pablo M. Lavados ${ }^{1}$, Arnold Hoppe $\mathbf{W}^{\mathbf{2}}$.}

${ }^{1}$ Jefe UTAC y Coordinador Programa Enfermedades Cerebrovasculares, Clínica Alemana de Santiago, Chile. Departamento de Ciencias Neurológicas, Facultad de Medicina, Campus Oriente, Instituto de Neurocirugía, Universidad de Chile. ${ }^{2} J e f e$ Servicio Neurología, Departamento de Medicina, Clínica Alemana de Santiago, Chile. 


\section{REFERENCIAS}

1. www.minsal.cl (observado en septiembre 2005).

2. Concha M, Aguilera X, Salas J. Estudio carga de enfermedad en Chile. MINSAL 1996. http:// epi.minsal.cl/epi/html/invest/ carga/nacional.htm (observado en octubre 2005).

3. Lavados P, Sacks C, Prina L, Escobar A, Tossi C, ARAYA F ET AL. Incidence, 30 days case fatality rate, and prognosis of stroke in Iquique, Chile: a 2 year community based prospective study (PISCIS project). Lancet 2005; 365: 2206-15.
4. Warlow CP, Sudlow C, Dennis M, Wardiaw J, Sandercock PAG. Stroke Seminar. Lancet 2003; 362: 1211-24.

5. Stroke Units Trialist' Collaboration (2005). A systematic review of specialists multidisciplinary team (stroke unit) care for stroke in patients. Stroke Module of Cochrane Database of systematic Reviews (3); 2005.

6. Meliado P, Court J, Godoy J, Mery V, Barnett C, ANDRESEN M ET AL. Características de la Enfermedad Cerebrovascular en un Neurointermedio Chileno: Análisis de 459 pacientes consecutivos. Rev Méd Chile 2005; 133: 1274-84. 